\title{
Rabdomiosarcoma Embrionario Uterino. Aspectos Morfológicos e Inmunohistoquímicos
}

\author{
Embryonal Rhabdomyosarcoma of the Corpus Uterine. \\ Morphological and Immunohistochemical Characteristics
}

Oscar Tapia E.

TAPIA, E. O. Rabdomiosarcoma embrionario uterino. Aspectos morfológicos e inmunohistoquímicos. Int. J. Morphol., 29(4):1126$1129,2011$.

RESUMEN: Los sarcomas uterinos son relativamente raros. El tipo histológico más frecuente es el leiomiosarcoma, seguido por el sarcoma del estroma endometrial. Los rabdomiosarcomas (RMS) son neoplasias malignas con diferenciación muscular esquelética. El rabdomiosarcoma embrionario (RMSE) tipo botrioide es el sarcoma más común de la infancia; con escasos reportes en adultos, afectando la región de cabeza y cuello, tracto genitourinario y extremidades más frecuentemente. En el tracto genitourinario, la vagina es la localización mas frecuente, comprometiendo en raras ocasiones el cuello o fondo uterino. Se presentan las características clínicas y morfológicas de un caso de RMSE uterino tipo botrioide diagnosticado en una paciente de 58 años en la Unidad de Anatomía Patológica del Hospital Hernán Henríquez Aravena de Temuco.

PALABRAS CLAVE: Rabdomiosarcoma embrionario; Rabdomiosarcoma; Sarcoma uterino.

\section{INTRODUCCIÓN}

Los sarcomas uterinos son relativamente raros. El tipo histológico más frecuente es el leiomiosarcoma, seguido por el sarcoma del estroma endometrial; un componente sarcomatoso puede también estar presente en el contexto de un tumor mixto Mülleriano maligno del útero, sin embargo, sarcomas uterinos puros distintos a los dos primeros mencionados son muy infrecuentes (McCluggage et al., 2002; Ober \& Tovell, 1959; Reynolds et al., 2006).

El rabdomiosarcoma en tanto es una neoplasia estromal con diferenciación muscular esquelética; siendo clasificados según la OMS como embrionario, alveolar o pleomórfico. El rabdomiosarcoma embrionario (RMSE) tipo botrioide por su parte, representa el sarcoma más común de la infancia; con escasos reportes en adultos, siendo la región de cabeza y cuello, tracto genitourinario y extremidades los sitios más comunmente afectados. En el tracto genitourinario, la vagina es la localización mas frecuente en población pediátrica, comprometiendo en raras ocasiones el cuello o fondo uterino de mujeres mayores (Hahlin et al., 1998; Hart \& Craig, 1978; Koukourakis et al., 2009; McCluggage et al.; Lloyd et al., 1983; Takano et al., 1999).
Se presentan las características clínicas y morfológicas de un caso de RMSE uterino tipo botrioide diagnosticado en una paciente de 58 años en la Unidad de Anatomía Patológica del Hospital Hernán Henríquez Aravena de Temuco.

\section{CASO CLÍNICO}

Mujer de 58 años, menarquia a los doce años, dos partos primero a los 31 años, tiempo total de lactancia aproximado de 20 meses, menopausia a los 47 años. Derivada desde centro de atención primaria por sangrado vaginal de 7 dias de evolución. El examen ginecológico evidenció sangrado activo por orificio cervical externo con protrusión de lesión polipoídea de 3 x 1,8 cm. La palpación bimanual mostró útero levemente aumentado de tamaño, sin lesiones anexiales. Se realizó extirpación por torsión de lesión descrita. La ecotomografía transvaginal demostró un útero de 45 x $65 \mathrm{~mm}$ de diámetro antero-posterior y transverso respectivamente, con engrosamiento endometrial irregular de 
hasta $21 \mathrm{~mm}$ y anexos uterinos de estructura conservada. Se envió para examen anatomo-patológico fragmentos disgregados del pólipo que en conjunto medían 2 x $1 \mathrm{~cm}$, de consistencia blanda en partes mixoídeo. Al examen microscópico, se reconocen fragmentos de pólipo endometrial con proliferación neoplásica estromal conformada por células que presentan núcleos de tamaño variable, hipercromáticos, redondeados a poligonales, dispuestos predominantemente bajo el epitelio endometrial superficial y alrededor de glándulas (Fig. 1A-B) con otras zonas hipocelulares de aspecto
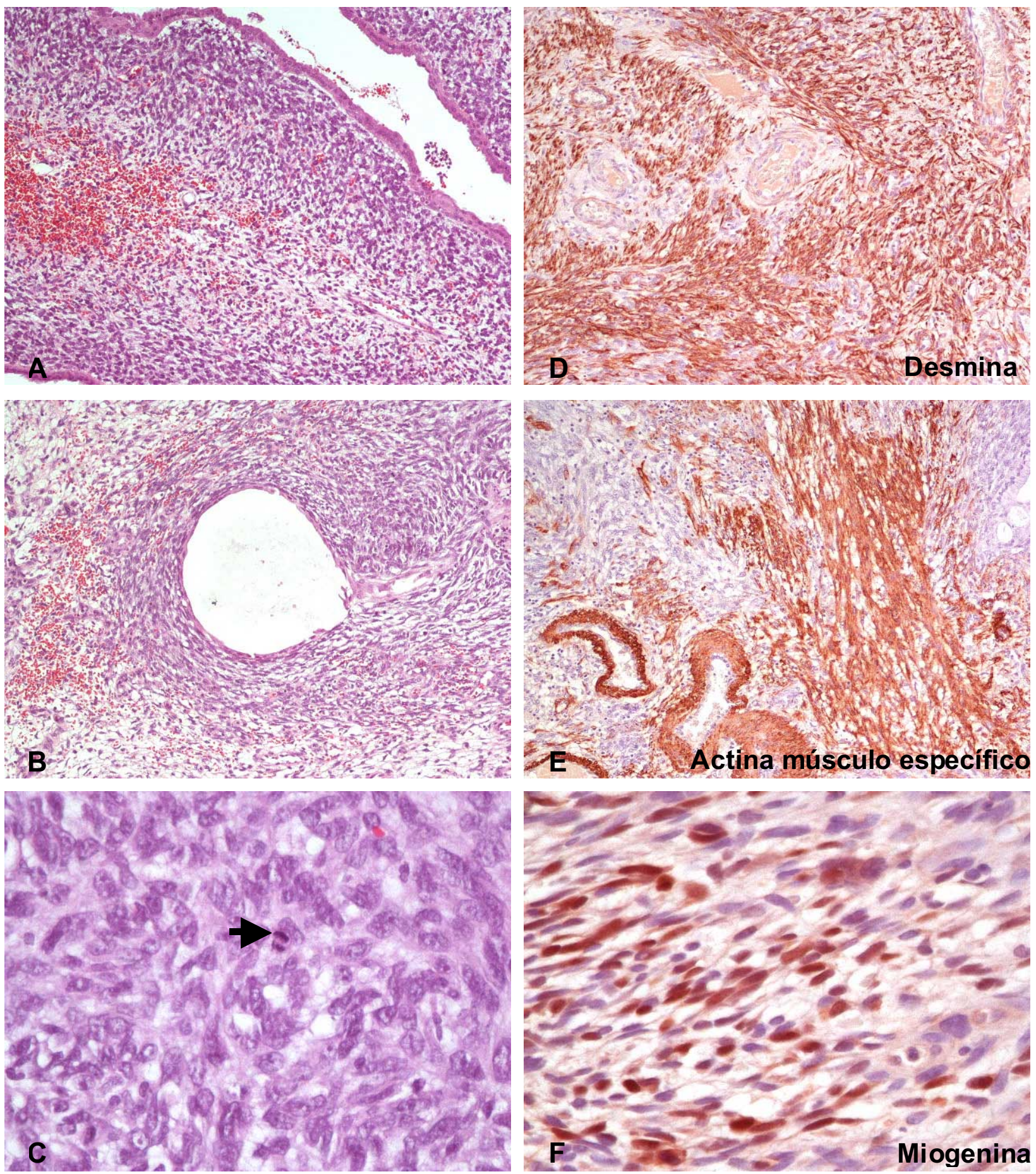

Fig. 1. A-B (HE, 100x). Proliferación neoplásica estromal con células predominantemente dispuestas bajo el epitelio (A) y rodeando glándulas (B). C (HE, 400x). Neoplasia compuesta por núcleos pleomórficos, hipercromáticos, poligonales, con cromatina vesiculosa y algunas mitosis (flecha). D-F, estudio inmunohistoquímico positivo para desmina, actina músculo específico y miogenina. 
edematoso o mixoídeo, y abundantes mitosis (10-15/10 campos de aumento seco mayor) (Fig. 1C). El estudio inmunohistoquímico mostró positividad de las células tumorales para desmina (Fig. 1D), actina músculo específico (Fig. 1E) y mioglobina (Fig. 1F). Con estos hallazgos morfológicos e inmunohistoquímicos se diagnosticó un rabdomiosarcoma embrionario tipo botrioide.

\section{DISCUSIÓN}

El RMSE es un tumor maligno originado en las células musculares embrionarias. Originalmente fueron clasificados como variantes de tumores Müllerianos mixtos malignos, sin embargo, actualmente es considerado una neoplasia heteróloga pura; en ausencia de un componente carcinomatoso. Este tumor es el sarcoma más común de la infancia y representa entre el 4 - $6 \%$ de todas las neoplasias malignas en este grupo etáreo, con escasos reportes en población adulta; siendo las primeras descripciones realizadas en 1854 por Weber (Atlante et al., 2000; Behtash et al., 2003; Beverly et al., 1993; Kempson \& Bari, 1970; Koukourakis et al.; Mousavi \& Akhavan, 2010; Takano et al.).

Los RMSE uterinos tipo botrioide presentan un patrón de crecimiento polipoídeo, en forma de racimo de uvas, con una consistencia gelatinosa, friable y que se desprende en fragmentos, produciendo frecuentemente hemorragias. Histológicamente está conformado por una población variable de células tumorales, algunas pequeñas, redondeadas con núcleos hipercromáticos, otras grandes, poligonales, con abundante citoplasma que se agrupan típicamente debajo de los epitelios o rodeando vasos sanguíneos y glándulas, alternando estas zonas de alta densidad celular con áreas de aspecto edematoso o mixoídeo, pudiendo observarse rabdomioblastos con estriaciones transversales. El recuento mitótico en tanto es elevado (Mousavi \& Akhavan; Palazzo et al., 1993; Takano et al.).

En estas lesiones el apoyo diagnóstico con inmunohistoquímica es de vital importancia, mostrando positividad para mioglobina y MyoD1 (marcadores de músculo esquelético), y desmina y actina músculo específico (HFF35) (marcadores de músculo liso y esquelético); siendo en tanto negativos para actina de músculo liso. Estos hallazgos proporcionan una prueba inequívoca de la diferenciación muscular esquelética al momento de hacer el diagnóstico (Koukourakis et al.; McCluggage et al.; Reynolds et al.; Takano et al.).

El pronóstico de esta enfermedad esta determinado por variables como el tamaño tumoral, ógano comprometido, edad del paciente, resultado quirúrgico (R0/R1) y presencia de metástasis. De esta manera niños con tumores localizados en vía biliar, vagina y región de la cabeza y cuello, menores de $5 \mathrm{~cm}$. de diámetro y que no presentan metástasis ganglionares y/o viscerales tendrían mejores tasas de supervivencias (Crist et al., 1995; La Quaglia et al., 1994; Lawrence et al., 1997; Smith et al., 2001; Spunt et al., 2000).

Por otro lado, el tratamiento de estos tumores considera la cirugía con criterio oncológico, variando esta de acuerdo al órgano afectado, seguida de quimioterapia, incluyendo radioterapia en caso de enfermedad residual (Koukourakis et al.; Mousavi \& Akhavan; Daya \& Scully, 1988).

Finalmente, el diagnóstico diferencial de esta neoplasia debe hacerse con pólipos fibroepiteliales en el cual existe una proliferación polipoide de tejido conectivo subepitelial en donde se pueden apreciar células de tipo fibroblástico hipertrofiadas que adquieren una morfología "rabdomioblástica", sin embargo no existe anaplasia, mitosis ni aglomeración celular sub epitelial, sin positividad para marcadores musculares al estudio inmunohistoquímico. Otras condiciones que deben descartarse son las neoplasias mixtas müllerianas, las que junto al componente carcinomatoso pueden presentar componentes sarcomatosos heterólogos entre ellos rabdomiosarcoma, debiendo por tanto examinarse cuidadosamente y mediante múltiples muestras para descartar la presencia de componente neoplásico epitelial.

TAPIA, E. O. Embryonal rhabdomyosarcoma of the corpus uterine. Morphological and immunohistochemical characteristics. Int. J. Morphol., 29(4):1126-1129, 2011.

SUMMARY: Uterine sarcomas are relatively rare. The most common histological type is leiomyosarcoma, followed by endometrial stromal sarcoma. The rhabdomyosarcoma (RMS) are malignant neoplasms with skeletal muscle differentiation. Embryonal rhabdomyosarcoma (RMSE) type botryoides is the most common in childhood, with few reports in adults, affecting the head and neck region, genitourinary tract and extremities more frequently. In the genitourinary tract, the vagina is the most common location, rarely involving cervix and fundus uterine. Clinical and morphological characteristics are presented of a case of uterine RMSE diagnosed in a 58 year-old woman in the Pathology Unit of the Hernán Henríquez Aravena Hospital in Temuco.

KEY WORDS: Embryonal rhabdomyosarcoma; Rhabdomyosarcoma; Uterine sarcoma. 


\section{REFERENCIAS BIBLIOGRÁFICAS}

Atlante, M.; Dionisi, B.; Cioni, M.; Di Ruzza, D.; Sedati, P. \& Mariani, L. Sarcoma botryoides of the uterine cervix in a young woman: a case report. Eur. J. Gynaecol. Oncol., 21(5):504-6, 2000 .

Behtash, N.; Mousavi, A.; Tehranian, A.; Khanafshar, N. \& Hanjani, P. Embryonal rhabdomyosarcoma of the uterine cervix: case report and review of the literature. Gynecol. Oncol., 91(2):452$5,2003$.

Beverly, R. J.; Hays, D. M. \& Tefft, M. Rhabdomyosarcoma. En: Pizzo, P. A. \& Poplack, D. G. (Ed.). Principles and Practice of Pediatric Oncology. $2^{\text {nd }}$ ed. Philadelphia, J. B. Lippicott Company, 1993. pp.769-71.

Crist, W.; Gehan, E. A.; Ragab, A. H.; Dickman, P. S.; Donaldson, S. S.; Fryer, C.; Hammond, D.; Hays, D. M.; Herrmann, J.; Heyn, R.; et al. The Third Intergroup Rhabdomyosarcoma Study. J. Clin. Oncol., 3(3):610-30, 1995.

Daya, D. A. \& Scully, R. E. Sarcoma botryoides of the uterine cervix in young women: a clinicopathological study of $13 \mathrm{ca}-$ ses. Gynecol. Oncol., 29(3):290-304, 1988.

Hahlin, M.; Jaworski, R. C.; Wain, G. V.; Harnett, P. R.; Neesham, D. \& Bull, C. Integrated multimodality therapy for embryonal rhabdomyosarcoma of the lower genital tract in postpubertal females. Gynecol. Oncol., 70(1):141-6, 1998.

Hart, W. R. \& Craig, J. R. Rhabdomyosarcomas of the uterus. Am. J. Clin. Pathol., 70(2):217-23, 1978.

Kempson, R. L. \& Bari, W. Uterine sarcomas: classification, diagnosis, and prognosis. Hum. Pathol., 1(3):331-49, 1970.

Koukourakis, G. V.; Kouloulias, V.; Zacharias, G.; Maravelis, G.; Papadimitriou, C.; Platoni, K. \& Gouliamos, A. Embryonal rhabdomyosarcoma of the uterine cervix. Clin. Transl. Oncol., 11(6):399-402, 2009.

La Quaglia, M. P.; Heller, G.; Ghavimi, F.; Casper, E. S.; Vlamis, V.; Hajdu, S. \& Brennan, M. F. The effect of age at diagnosis on outome in rhabdomyosarcoma. Cancer, 73(1):109-17, 1994.

Lawrence, W. Jr.; Anderson, J. R.; Gehan, E. A. \& Maurer, H. Pretreatment TNM staging of childhood rhabdomyosarcoma: a report of the intergroup rhabdomyosarcoma study group. Children's cancer study group. Pediatric oncology group. Cancer, 80(6):1165-70, 1997.

Lloyd, R. V.; Hajdu, S. I. \& Knapper, W. H. Embryonal rhabdomyosarcoma in adults. Cancer, 51(3):557-65, 1983.

McCluggage, W. G.; Lioe, T. F.; McClelland, H. R. \& Lamki, H. Rhabdomyosarcoma of the uterus: report of two cases, including one of the spindle cell variant. Int. J. Gynecol. Cancer, 12(1):128-32, 2002.

Mousavi, A. \& Akhavan, S. Sarcoma botryoides (embryonal rhabdomyosarcoma) of the uterine cervix in sisters. J. Gynecol. Oncol., 21(4):273-5, 2010.

Ober, W. B. \& Tovell, H. M. Mesenchymal sarcomas of the uterus. Am. J. Obstet. Gynecol., 77(2):246-68, 1959.

Palazzo, J. P.; Gibas, Z.; Dunton, C. J. \& Talerman, A. Cytogenetic study of botryoid rhabdomyosarcoma of the uterine cervix. Virchows Arch. A Pathol. Anat. Histopathol., 422(1):87-91, 1993.

Reynolds, E. A.; Logani, S.; Moller, K. \& Horowitz, I. R. Embryonal rhabdomyosarcoma of the uterus in a postmenopausal woman. Case report and review of the literature. Gynecol. Oncol., 103(2):736-9, 2006.

Smith, L. M.; Anderson, J. R.; Qualman, S. J.; Crist, W. M.; Paidas, C. N.; Teot, L. A.; Pappo, A. S.; Link, M. P.; Grier, H. E.; Wiener, E. S.; Breneman, J. C.; Raney, R. B.; Maurer, H. M. \& Donaldson, S. S. Which patients with microscopic disease and rhabdomyosarcoma experience relapse after therapy? A report from the soft tissue sarcoma committee of the children's oncology group. J. Clin. Oncol., 19(20):4058-64, 2001.

Spunt, S. L.; Lobe, T. E.; Pappo, A. S.; Parham, D. M.; Wharam, M. D. Jr.; Arndt, C.; Anderson, J. R.; Crist, W. M.; Paidas, C.; Wiener, E.; Andrassy, R. J. \& Schwartz, C. L. Aggressive surgery is unwarranted for biliary tract rhabdomyosarcoma. $J$. Pediatr. Surg., 35(2):309-16, 2000.

Takano, M.; Kikuchi, Y.; Aida, S.; Sato, K. \& Nagata, I. Embryonal rhabdomyosarcoma of the uterine corpus in a 76-year-old patient. Gynecol. Oncol., 75(3):490-4, 1999.

\author{
Dirección de correspondencia: \\ Dr. Oscar Tapia E. \\ Departamento de Anatomía Patológica \\ Facultad de Medicina \\ Universidad de La Frontera \\ Manuel Montt 112 \\ Código Postal 478-1176 \\ Temuco \\ CHILE
}

Fono: $045-296530$

Email: otescalona@gmail.com

Recibido : 23-06-2011

Aceptado: 28-09-2011 